\title{
SOME INTERESTING FACTS CONCERNING THE X-RAY
}

\author{
BY JAMES H. PROTHERO, D.D.S., Chicago, Illinois
}

(Read before the Illinois State Dental Society, Springfield, Illinois, May 9-11, 1922)

$\mathrm{A}^{\mathrm{n}}$ MONG the many remarkable scientific advances within the last thirty years, none are more interesting and truly wonderful than the discovery of the X-ray. The story of how nearly scientists came at different times to finding it, and yet failed, and how by accident it was discovered, is very entertaining.

As a matter of fact the development of electrical science from the very first experiment with amber down to the latest developments in the radio field, has been replete with unique surprises.

Nearly three thousand years ago someone discovered that by rubbing a bit of amber with silk, it would pick up small pieces of straw and other light substances. The Ancients believed that the curiously shaped brownish pieces of this material found along the shores of the Baltic were Jupiter's congealed thunderbolts, or in other words, the end product of lightning. This was a close guess for we now realize that the attraction exhibited by these bits of mineralized resin is identical with that force which Franklin brought down from the clouds with his "kite and bottle and key."

For nearly two thousand years this classic experiment apparently satisfied the curious, as no records exist of any further efforts to determine whether other substances possessed this property.

In 1570 Dr. William Gilbert discovered that glass, sulphur and other kinds of resin, when energized by friction, would behave in a manner similar to amber. Crude frictional machines were soon devised for generating larger quantities of the newly discovered force, and many interesting experiments are cred- ited to Gilbert and his co-laborers. The Greek name for amber is electron, and to commemorate the ancient experiment Gilbert called the force with which he was experimenting, "electricity," which name it bears today.

The work of Gilbert, Gray, Franklin, Coulomb, Galvani, Volta, Davy, and many others, although not particularly correlated at the time of their experiments, constitutes the preliminary basis of modern electrical science.

Contributing particularly to the line of experiments which resulted in the discovery of the X-ray may be mentioned Faraday, Clerk Maxwell, Geissler, Crookes, Hertz, Lenard, and Roentgen. Others have given much also, but these deserve special credit for having devised essential apparatus and carried forward original investigations of great value.

Faraday was probably the first to use a partial vacuum tube and experiment with electric discharges in rarefied gases, while his studies in electro-magnetic induction led to the development of the dynamo.

Clerk Maxwell, in 1866, demonstrated mathematically that electrical disturbances ought to develop waves in the ether, that intangible substance which pervades all space.

Heinrich Hertz, in 1888, demonstrated experimentally, the truth of Maxwell's mathematical proposition. Hertz devised an apparatus called an oscillator consisting of an induction coil arranged with metal ball terminals so related as to form a spark gap. In action, when sufficient difference of potential had been established to overcome the resistance between the dielectric or air between the terminals, 
sparks jumped the gap and developed in the ether, waves which passed out in all directions, but which manifested themselves more strongly in a direction at right angles to the spark gap.

These were called electro-magnetic waves. To detect them Hertz devised a very simple but effective appliance of wire, bent in the form of an open ring and having ball terminals, thus forming a spark gap.

When held so that the ether waves passed through the ring, the wire vibrated and sparks jumped the gap. This was called a resonator. The effect of the oscillator upon the resonator when a current passes has been likened to what occurs when two tuning forks of the same pitch are placed some distance apart and one is caused to vibrate. The ether waves which in this case conduct sound, set up corresponding vibrations in the inert fork.

It is this principle of generating electro-magnetic waves which forms the basis of present day wireless telegraphy. The distance between the crests of long distance wireless waves is frequently 15,000 meters or about nine miles. These electro-magnetic waves travel with the speed of light or 186,300 miles per second. If sufficient energy could be imparted, they would circle the globe seven and onehalf times in one second.

In 1869 , Geissler devised the first permanent vacuum tube and succeeded in rarefying it to a much higher degree than had previously been possible. Heretofore, in all the experiments with rarefied tubes, from Faraday's observations down to and including the work of Geissler, the vari-colored luminosity within the apparatus, when energized, was of greater interest. This luminosity originated at the anode and extending toward the cathode partially filled the tube, presenting purple, pink, green, yellow and gray tinges depending on the degree of rarefaction of the tube and the character of the contained gases. On raising the vacuum of the tube to a high degree, the anode luminosity disappeared and from the cathode there was projected toward the anode a faint line illumination, subsequently called the cathode stream.

In 1879, Crookes modified the Geissler tube by inserting a third terminal in the middle of the glass cylinder to serve as a cathode while the two end terminals were connected to form the anode. With this as well as with the Geissler tube he performed many varied and interesting experiments. He demonstrated that the cathode emitted some sort of radiation, that it cast a shadow of an opaque object on the tube walls, that it would revolve a vane placed within its path and that it could be deflected by a magnet applied from without.

$\mathrm{He}$ modified the flat cathode terminal giving it a concave form which served to concentrate the cathode stream at a focal point. By locating the anode at this point it became intensely heated.

These observations led him to believe that the cathode stream consisted of negatively charged particles of matter in a minute state of subdivision, a deduction which has since been verified.

One other experiment of Crookes deserves special mention. On raising the vacuum of the tube to a high degree he observed that at a point opposite the dark cathode end, on the inner surface of the glass, a green patch appeared which afterward was shown to be the source of the X-ray.

In 1892, Hertz demonstrated that the cathode stream would pass through gold leaf inserted in its path within the tube. Experiments with silver and aluminum foils showed similar results. These observations led him to believe that since the cathode stream could pass through metals within the tube, something must occur outside the walls. Crookes had previously endeavored to bring the stream outside the tube but was unsuccessful.

Before Hertz could accomplish anything in this prospective field, death ended his most useful and interesting career. His work, and particularly this latest suggestion, was continued with en- 
thusiastic zeal by Lenard, his pupil and co-worker.

Since in Hertz's latest experiment the cathode stream passed through aluminum foil within the tube, Lenard constructed one in which an aluminum window was set in the tube wall at the point of focus of the cathode steam. When energized, he found that the stream passed through the metal window, that it caused phosphorescence of uranium, that the stream could be deflected by a magnet, and that it possessed actinic properties as shown by its action on photographic plates. $\mathrm{He}$ further observed that the higher the vacuum, the more penetrative became the external cathode rays.

That he was working with X-rays, but failed to recognize them, is now a generally conceded fact. The main point which Lenard and all experimenters before him failed to observe, but which Roentgen recognized was that when cathode rays strike matter they produce a different kind of radiation.

In 1895, Roentgen who had been interested in Lenard's work, was one day experimenting with a Crookes' tube. His attention being diverted from his work for a short time, he without design laid the energized tube on a book which contained a flat key used as a book mark. Beneath the book and in a plate holder was an unexposed photographic plate. Later the plate was used, with others in outdoor photography and developed in the usual manner. One of them showed the shadow of the key which he recognized, but could not account for its presence.

In the Lenard tube with its aluminum window, it had been demonstrated that the cathode rays passed out through the metal. The tube used by Roentgen was a glass walled tube with no such means of exit for the rays.

It was thought by Roentgen, and others, that nothing could pass through the glass, yet something within or outside the tube had caused the image of the key to appear on the plate. To prove or disprove this theory, the book containing the key was again placed upon an unexposed plate, the tube placed upon the book and energized, and the plate developed. 'The results were as surprising to Roentgen, as it was shortly after to the whole world, for the shadow of the key was on the plate.

Various other experiments were conducted which led him to the conclusion that a ray invisible to the eye, but capable of penetrating opaque substances emanated from the tube. He then placed his hand upon an enclosed photographic plate, developed the cathode stream in a Crookes' tube in close proximity to it, and secured a radiograph of the bones. $\mathrm{He}$ further found that the emanations from the cathode stream caused fluorescence in a cardboard coated with platino-barium cyanide, not only on the side presented to the tube, but on the reverse side as well. This led to the experiment of placing his hand between the screen and the tube with the result that on the reverse side the shadow of the bones was clearly outlined.

Roentgen's discovery made a most profound impression, not only in scientific circles, but throughout the world. Here was a new kind of ray or light, hitherto unsuspected, which although beyond the visibility of human eye, could leave its record on a photographic plate. Not only that, but by means of suitable apparatus, one could virtually look into the human body, discover fraciures and dislocations of bones, locate foreign bodies, and bring into view pathologic conditions not otherwise recognizable.

This invisible radiation, the existence of which was apparent only by its results, was puzzling in the extreme. It was an unknown quantity and in accordance with algebraic custom Roentgen named it the X-ray. It is frequently called the Roentgen Ray in honor of its discoverer, but the first name given it seems quite appropriate and is in general use. 
Present Methods of X-ray ProducTION

Various radiographic equipments are in use at this time, description of which is unnecessary further than to say that by means of induction coils, motor-generators, transformers, etc., a current of high voltage, low amperage, and unidirectional in character is readily developed for passage through the $\mathrm{X}$-ray tube.

The tubes used at present are of two distinct types; first the gas tube which although reduced to a comparatively high vacuum, still contains a small amount of air or gas, the purpose of which will be shown later.

Second, a type known as the Coolidge tube, so-called from its inventor, and which is reduced to as nearly a perfect vacuum as human skill can produce. It is said that this tube contains less than one millionth its cubic content of air or gas.

These tubes which resemble each other in general form consist of a sphere of glass from three to seven inches in diameter, having two axial extensions, opposite each other, through which wires pass to the interior terminals.

The gas tubes, have, in addition to the extensions mentioned, one and sometimes two other projections, carrying fixtures for raising or lowering the vacuum and for taking care of the overload.

Projecting inwardly from one of the axial tubes is a rod which terminates in the center of the sphere in a large short cylinder, the end of which is bevelled at an angle of 45 degrees to the long axis of the tube. This is called the anode or positive pole. Through the opposite axial arm projects another metal wire and rod which terminates within a short distance of the anode. This presents a concave surface toward the anode and is called the cathode or negative pole.

The tube when placed in a suitable holder and properly connected is energized by passing through it, a high tension current of from 10,000 to 100,000 volts and from 10 to 60 or more milliamperes, depending on the requirements.
The discharge from the cathode to the anode consists of a stream of negatively charged electrons, estimated to be $1 / 1800$ the mass of a hydrogen atom. These electrons are the result of the disruption of gas atoms in a highly rarefied gas tube, while in a Coolidge tube, practically devoid of gas they are supplied by the incandescent filament of tungsten.

Their velocity is estimated at $1 / 30$ to $1 / 3$ that of light, or from 6210 to 62100 miles per second, varying with the strength of current.

These electrons serve as conductors for the current since the latter cannot, without some such medium, jump the gap in a vacuum.

Although invisible to the eye, and almost inconceivable as to mass, the terrific speed at which they are driven across the gap, results in the development of intense heat in the center of the anode. To avoid fusion of the latter, the central portion is made of platinum, or tungsten.

The point of contact or focus of the cathode stream against the anode is the site of development of the X-ray, which as it is formed radiates outward in all directions, but particularly from the active hemisphere.

Physicists believe that the sudden stopping or impact of the swiftly moving electrons against the anode and each other is the direct cause of X-ray emanations. These rays after their exit from the tuhe, set up transverse etheric vibrations, the wave length of which cannot be measured by the usual diffraction grating. They can, however, be estimated by passing them through a crystal. This has been done, the results of which show them to be about $1 / 10,000$ that of light waves or about .014 of a micron.

Accurate calculations have shown that the etheric vibrations of X-rays are the shortest so far discovered. This places them at the lower end of the spectrum, beyond the violet. They are in fact known as the tri-ultra violet rays. Similar calculations in reference to long dis- 
tance etheric waves places them at the upper end of the spectrum, or far beycnd the red. Further, it has been demonstrated that light rays, X-rays and the gamma rays of radium are alike except in wave lengths.

Briefly stated, the development of the $\mathrm{X}$-ray is accomplished by the separation of electrons from molecules, their projection at high speed, and their sudden arrest of motion.

\section{IMPORTANT CHARACTERISTICS}

The principle characteristics of the $\mathrm{X}$-ray to be considered have been classified by Hirsch as follows:

1. Their effect on photographic plates.

2. The development of fluorescence in certain substances.

3. Power of penetrating opaque substances.

4. Action on living tissue.

5. Power of causing chemical reaction.

6. Property of ionizing gases.

Brief mention only can be made of some of these characteristics. The two factors, the power of acting on photographic plates and of penetrating opaque substances are particularly interesting. In regard to photographic properties, it is believed that when either light or the $\mathrm{X}$-ray is directed upon a molecule of silver salt an electron from the latter is set free. This disturbance of the molecular cohesion is sufficient to render the entire molecule developable. The penetrability of opaque substances by the Xray is believed to be due to its diminutive wave length.

Fluorescence has been defined as "A phenomenon in which radiant energy of one wave length is absorbed by a material and emitted as radiant energy of a longer wave length." This property of the X-ray is made use of in fluoroscopic examination of the living subject, and by means of intensifying screens in radiographic technic for accellerating the speed of exposure.
Since X-rays are developed within a very small area, at the point of concentration of the cathode stream against the target, and the emanation radiates out in all directions in straight lines, it becomes a difficult problem to secure shadow studies of accurate mathematical dimensions, of objects interposed in their path.

A tube placed close to the object radiographed will result in an enlarged shadow on the film. A film placed a considerable distance from the object will result, not only in an enlarged shadow on the film, but the details and the shadow itself will be more or less indefinite.

A film placed at other than a right angle to the energetic central ray will usually result in a distorted image. A shadow projected on a curved film will be more or less disproportioned.

Penetrative power of X-rays decreases in inverse ratio to distance of target from object.

When primary rays strike dense objects, secondary radiations are developed, which, although traveling in straight lines assume angles at variance to the rays from which they originate. These secondary rays are a detriment as they tend to fog the film and render the shadow more or less indefinite.

Time, penetrative power of the rays, distance, and relative position of tube, object and film are important factors which must be correlated in order to produce good radiographs.

The effect of the X-ray on living tissue may be soothingly stimulating or insidiously destructive, depending on conditions under which applied. When judiciously used, in selected cases, it is a most valuable curative agent. Lupus Vulgaris, rodent ulcer, certain types of malignant tumors and some superficial infections are frequently benefitted and sometimes eradicated by its application.

Some of the ill-effects resulting from undue exposure to the X-ray are as follows: Applied indiscreetly it will cause a condition similar to sunburn. Con- 
tinued exposure results in dermititis, acute at first which may disappear if application of rays is discontinued, but chronic if exposures are prolonged. Temporary loss of hair is not uncommon but if continued lengthy exposures are made permanent baldness may result, due to destruction of the hair follicles. Pigmentation of the skin and splitting as well as entire loss of the finger nails are occasionally recorded.

Radiographers who constantly work in the presence of the ray without adequate protection are sometimes troubled with the development of horny excrescences on the hands and face. This condition frequently persists long after exposures to the rays have ceased. In some cases epithelioma and various other malignant tumors attributable to X-ray burns have been reported.

G. Contramoulins, Chief of Radiographic Laboratories, Paris, after twenty years of careful effort on his part to avoid injury reports that up to the war he had apparently succeeded, but imperfect protection while searching for foreign bodies, had brought on the disaster he so feared and he predicts that he shall end in the manner of his late associates.

The discovery of this mild but dangerous force of Nature has resulted in alleviating human suffering, and prolonging life. It has lightened the burdens of those engaged in the healing art to an immeasurable degree, particularly in the surgical field, where, with his eyes, the operator can as previously stated examine obscure wounds, fractures, dislocations and locate foreign bodies, and beforehand determine the best method of procedure.

In the field of physical science the $\mathrm{X}$-ray has brought to light many hidden and wonderful secrets of nature. So much has been discovered by its aid that the physicist has found it necessary to revise his theories and reconstruct his hypotheses.

To illustrate this last statement and show wherein the $\mathrm{X}$-ray has played an important part, we will revert to a few things which occurred about the time Roentgen made the great discovery.

In 1894, Rayleigh discovered in the atmosphere a new gaseous element which was named helium. Soon after Ramsey found four other elements, viz: neon, argon, krypton, and xenon, all possessing somewhat similar properties, all incapable of entering into chemical combination with other elements except at white heat. The discovery of radium emanations by Bequerel almost immediately followed. Shortly after the Curie's isolated the chloride and bromide of radium. This element has a high atomic weight and possesses the property of decomposing silver salts similarly to the X-ray.

Shortly after Madame Curie discovered that radium broke down or decayed into a gas. Within four days from the formation of its salt half of it decomposed, but during that time it evolved three million times more energy proportionately than was developed from any known chemical action. This gas decomposed into helium, the elemental gas discovered by Rayleigh.

In an effort to utilize some of the enormous energy resulting from the decay of the radium emanation, Ramsey placed it in water. Instead of helium showing as an end product, neon appeared, the second of the elemental gases named. Copper sulphate was then added to the water and instead of neon showing, as an end product, argon appeared.

In this same experiment the water was partially decomposed into its constituent gases, not in the usual proportion however, but with a decided percentage loss of oxygen and a marked percentage increase of hydrogen. Further there was a loss of copper, and lithium appeared where none had been present before. The deductions were that through the influence of radio-activity something had been changed into hydrogen and copper had been degraded into lithium.

Professor J. J. Thompson has shown that in the energetic field of an X-ray tube many substances give off particles 
charged with positive electricity, that these particles are independent of and differ in character from the gas from which they originate, that they are of two kinds - one to all appearance identical with the hydrogen atom, the other resembling in every respect the alpha particles which emanate from radio-active substances.

These experiments of Thompson's conducted in the X-ray field without employing any radio-active substances proves the work of Ramsey and others and establishes the fact that hydrogen results from the debasement of other elements under favorable conditions.

It is evident then, from these and many other check up experiments that uranium is transmuted into radium, that radium may be transmuted into helium, neon or argon, that copper under the influence of the alpha rays may be reduced to lithium, that many inert substances break down into hydrogen when subjected to the energizing action of the X-rays and alpha corpuscles, and finally that the radium emanations are themselves the product and evidence of elemental decay.

These facts which have been worked out time and again by the world's greatest scientists form the basis of the new physics, and in this new science the X-ray has played a most important part.

The theory of the unity or oneness of matter is very old. It has been repeatedly advanced since Lucretius' time two thousand years ago, and as repeatedly scoffed at and denounced as senseless, yet in the light of radium degradation and X-ray revelations, there is a growing belief that it may prove true.

Professor J. J. Thompson has expressed the opinion that all of the elements represent successive condensations of one primary stuff whose atoms called electrons or corpuscles weigh less than the $1 / 1000$ part of the lightest known terrestrial element, namely hydrogen. This primary stuff is negative electricity which is therefore a true chemical element. 